\title{
CDK5/CAMK2-DRP1-AMPK-Mediated Mitochondrial Fission is Required for Imatinib Resistance of CML Cells
}

\section{Ziyuan Lu ( $\sim$ Lzo19880306@126.com )}

Southern Medical University Nanfang Hospital

Jiaming Tang

Third Affiliated Hospital of Guangzhou Medical College

\section{Yuling Li}

Third Affiliated Hospital of Guangzhou Medical College

\section{Guohua Liang}

Third Affiliated Hospital of Guangzhou Medical College

\section{Xiaoyun Chen}

Third Affiliated Hospital of Guangzhou Medical College

\section{Bing Luo}

Third Affiliated Hospital of Guangzhou Medical College

\section{Qian Qu}

Third Affiliated Hospital of Guangzhou Medical College

\section{Research}

Keywords: DRP1, Mitochondrial fission, Autophagy, Apoptosis, Energy metabolism, Chronic myeloid leukemia, Drug resistance

Posted Date: August 6th, 2020

DOI: https://doi.org/10.21203/rs.3.rs-52123/v1

License: (c) (1) This work is licensed under a Creative Commons Attribution 4.0 International License. Read Full License 


\section{Abstract}

Background: In chronic myeloid leukemia (CML), resistance to tyrosine kinase inhibitors (TKIs) is still a serious clinical challenge, especially in the context of multi-resistance BCR-ABL mutations, such as T315I. Increasing evidence has demonstrated that mitochondrial fission plays an important role in cancer stem cell maintenance and drug resistance. Therefore, as a vital fission-related protein, DRP1 serves as a novel target for cancer treatment. However, whether DRP1 inhibition could overcome imatinib resistance in CML remains unknown.

Methods: We used bioinformatic analysis and a confocal microscopy experimental approach to investigate whether mitochondrial fission could be involved in drug resistance in CML. Next, we applied biological and metabolic detection methods to reveal the roles of DPR1 in autophagy, apoptosis, and mitochondrial respiration in CML cells and determine whether coupling imatinib with DRP1 inhibition has a synergetic anti-leukemic effect on both drug-sensitive and drug-resistant CML cells.

Results: Resistant CML cells displayed fragmented mitochondria and were accompanied by overexpression of active DRP1 compared with their sensitive counterparts. Furthermore, blocking DRP1 decreased mitochondrial respiration via the CDK5/CAMK2-DRP1-AMPK signaling pathway. Additionally, DRP1 inhibition perturbed the balance between mitochondrial-dependent apoptosis and autophagy, which led to cell death. Finally, combination treatment with imatinib and DRP1 inhibition exhibited a synergetic anti-leukemic effect on both drug-sensitive and drug-resistant CML cells (including CML cells carrying T315I mutation) in in vitro or in vivo experiments.

Conclusions: Combination treatment with imatinib and DRP1 inhibition may represent a promising strategy to overcome CML drug resistance.

\section{Background}

Chronic myeloid leukemia (CML) is a malignant myeloproliferative disorder caused by the clonal expansion of a transformed multipotent hematopoietic stem cell expressing BCR-ABL, a chimeric oncoprotein with constitutive tyrosine kinase activity. Tyrosine kinase activity promotes CML cell survival and proliferation and is insensitive to apoptosis [1]. The first tyrosine kinase inhibitor (TKI) designed, imatinib, is widely used to treat CML by selectively inhibiting BCR-ABL-positive CML cells as evidenced by in vitro and in vivo research data [2]. Despite the effective treatment of patients with $\mathrm{CML}$, prolonged treatment with TKIs (including second- and third-generation TKIs) to maintain remission is often linked to drug toxicity and/or acquired resistance, coupled with high economic costs [3].

Drug resistance in CML can be classified into two major groups: BCR-ABL-dependent and BCR-ABLindependent [4-9]. The most frequently identified mechanism of TKI resistance involves point mutations associated with the BCR-ABL kinase domain that involves TKI binding. There are more than 100 different mutations identified to date, and T315I is the most challenging because it confers resistance to imatinib 
this context, demonstrating activity against T315I mutant clones. However, ponatinib's safety data showed an increased risk of serious cardiovascular side effects in a dose-dependent manner [11]. Therefore, overcoming resistance to TKIs, especially T315I mutation-mediated resistance, is still a barrier to improving the survival of patients with CML.

Mitochondria are extremely dynamic, constantly changing their morphology to satisfy the requirements of cells and adapt to the cellular environment [12]. In most healthy mammalian cells, the mitochondrial network exists as mixed structures of long interconnected tubules with short isolated dot-like spheres, which are precisely regulated by dynamic remodeling via the balance between fusion and fission events. Mammalian GTPases mitofusins 1 and 2 (MFN1 and MFN2) are responsible for outer mitochondrial membrane fusion, while optic atrophy 1 (Opa1) mediates inner mitochondrial membrane fusion. Conversely, dynamin-related protein 1 (DRP1, or Dynamin-1-like protein, DNM1L) and FIS1 (fission, mitochondrial 1) are required for mitochondrial fission [13]. Mitochondrial dynamics serves to intermix the contents of the mitochondrial population, control the morphology of mitochondria, degrade damaged mitochondria, and maintain their high functionality. Recently, cumulative evidence has indicated the close links between cancers and unbalanced mitochondrial dynamics. This concept is further supported by multiple studies indicating enhanced mitochondrial fission and reduced fusion in some tumor cells [14]. In addition, DRP1 is a vital fission-related protein that contributes to stemness in glioblastoma [15], proliferation sustenance in lung cancer [16], cellular metabolism change in melanoma [17], as well as participates in the metastases of breast tumors [18]. Furthermore, reduced cancer growth and increased apoptosis induced by inhibiting DRP1 have been found in several cancer types [19]. Despite strong laboratory evidence emphasizing the key role of DRP1 in tumor biology, the underlying mechanism for this association is still being explored.

In the present study, we dissected mitochondrial fission in human CML with a focus on its cellular biology. We showed that DRP1 is overexpressed in imatinib-resistant CML cells. CDK5/CAMK2-DRP1AMPK regulates mitochondrial activity, which maintains CML resistance. Inhibition of DRP1 induces mitochondria-related apoptosis, reduced autophagy activity, decreases oxidative phosphorylation (OXPHOS) levels, and improves imatinib sensitivity in CML-resistant cells. These findings provide new perspectives for the treatment of CML.

\section{Methods}

\section{Bioinformatics analysis}

A microarray dataset (accession number GSE33290) was collected from the National Institutes of Health (NIH)-National Center for Biotechnology Information (NCBI)-GEO Dataset databases [20]. Expression data from imatinib treatment cytogenetic responders $(R)$ and non-responders (NR) with CML chronic phase were compared to identify significantly differentially expressed genes. Using the "cluster profiler" package of "R×64 3.4.1" software, differentially expressed genes were subjected to gene ontology (G0)-based 
enrichment analysis (biological process, cellular component, and molecular function) and Kyoto Encyclopedia of Genes and Genomes (KEGG) pathway enrichment analyses.

\section{Cell lines and cultures}

Human KBM5 cells, which are imatinib-sensitive cells, were purchased from the Cell Bank of the Chinese Academy of Sciences (Shanghai, China). KBM5 cells with T315I mutation (KBM5-STI cells), which were provided by Prof. Michael Andreeff (Section of Molecular Hematology and Therapy, Department of Leukemia, The University of Texas M.D. Anderson Cancer Center, Houston, TX, USA), were used as imatinib-resistant cells. The cells were cultured at $37^{\circ} \mathrm{C}$ and $5 \% \mathrm{CO}_{2}$ in complete media consisting of DMEM (Dulbecco's modified Eagle medium) (Gibco, Grand Island, NY, USA) supplemented with 10\% fetal bovine serum (Gibco), 100 units/mL of penicillin (Sigma, St. Louis, MO, USA), $100 \mu \mathrm{g} / \mathrm{mL}$ streptomycin, and $1 \%$ glutamine (Sigma).

\section{Vector construction}

The DRP1 knockdown lentiviral vector (Lv-DRP1-OE) and DRP1 overexpressing lentiviral vector (LvshDRP1) were provided by Guangzhou Land Bio Co, Lit (Guangzhou, China). The constructed vectors were confirmed by qPCR and western blotting. Finally, the expression and interference efficiency of the target genes were verified by RT-PCR.

\section{Chemicals}

Imatinib (IM, Cell Signaling Technology, Danvers, MA, USA ) was dissolved in DMSO at a stock concentration of $10 \mathrm{nM}$ and stored at $-20^{\circ} \mathrm{C}$. Mdivi-1 (Abcam, Cambridge, UK) was dissolved in DMSO (Cell Signaling Technology) at a concentration of $50 \mathrm{mM}$.

\section{Confocal microscopy}

Cells were seeded onto six-well plates and grown to 70\% confluence. MitoTracker-DeepRed dye (Invitrogen, California, USA) or autophagy detection reagent (Abcam) was used to label the mitochondria or autophagosomes of leukemic cells, at a final concentration of $250 \mathrm{nM}$, for $30 \mathrm{~min}$ at $37^{\circ} \mathrm{C}$ in a $5 \% \mathrm{CO}_{2}$ incubator before harvesting the cells for confocal imaging. Before placing onto slides, labeled cells were washed three times with ice-cold FACS buffer and three times with cold PBS (Gibco), and then suspended at a final concentration of $5 \times 10^{3}$ cells $/ \mathrm{mL}$ in ice-cold PBS. The slides were air-dried in the dark at $37^{\circ} \mathrm{C}$ for 5 min, fixed with $100 \%$ methanol (Sigma) at $-20^{\circ} \mathrm{C}$ for $10 \mathrm{~min}$, air-dried in the dark at $37^{\circ} \mathrm{C}$ for $5 \mathrm{~min}$, and mounted onto slides. The slides were imaged using an Olympus FV3000 confocal microscope (Olympus, Tokyo, Japan).

\section{Transmission electron microscopy}

The leukemic cells were stained with uranyl acetate and lead citrate on a microtome (Leica Ultracut; 
Tokyo, Japan) at an accelerating voltage of $60 \mathrm{kV}$. Micrographs were taken at $7000 \times$ or $20000 \times$ magnification. Quantification of mitochondrial volume was performed by drawing a contour of the crosssection of each mitochondrion and then calculating the area of the enclosed region using ImageJ software, version $1.50 \mathrm{i}$.

\section{Apoptotic analysis}

An apoptosis assay was performed using the annexin V-FITC and PI apoptosis detection kit (Dojingdo, Kumamoto, Japan) according to the manufacturer's protocol. After $48 \mathrm{~h}$ treatment with imatinib and/or Mdivi-1, the cells were analyzed using a FACSCalibur flow cytometer (BD Bioscience, San Jose, CA, USA).

\section{Protein lysate preparation and western blotting}

Cells were harvested and washed in ice-cold PBS before being lysed in 1× RIPA buffer. The RIPA-lysed samples were quantified using a BCA protein assay kit (ThermoFisher Scientific, Waltham, MA, USA). For western blotting, proteins were separated by sodium dodecyl sulfate polyacrylamide gel electrophoresis (SDS-PAGE), transferred onto PVDF membranes, and sequentially incubated overnight with primary antibodies at $4^{\circ} \mathrm{C}$. The PVDF membranes were then incubated with secondary antibodies at $37^{\circ} \mathrm{C}$ for $2 \mathrm{~h}$ and subjected to imaging. Before the analysis of DRP1, the cytosolic and mitochondrial sub-cellular fractions were separated using a mitochondria isolation kit (Beyotime Biotechnology, Shanghai, China). Western blotting was conducted using the following primary antibodies: rabbit anti-human DRP1, cytochrome c, Cleaved-Caspase-9, Cleaved-Caspase-3, p-AKT(Ser473), p-AKT(Thr308), mTOR(Ser2448), P62, LCI/II, CDK5, CAMK2, p-AMPK, and $\beta$-actin, which were obtained from Cell Signaling Technology.

\section{Oxygen consumption rate (OCR) measurements}

KBM5, KBM5-STI, KBM5-shDRP1, or KBM5-DRP1-overexpressing (KBM5-DRP1-OE) cells were seeded at a density of $3 \times 10^{5}$ cells per well on Seahorse Biosciences 24-well culture plates (Seahorse Bioscience, Shanghai, China) coated with Cell-Tak solution ( $1 \mathrm{mg} / \mathrm{mL}$, ACRO Biosystems, Newark, DE, USA) before the beginning of the assay. After serum shock synchronization, the cell medium was exchanged with 500 $\mu \mathrm{L}$ of the testing medium [glucose-free RPMl-1640 medium (Gibco) containing $2 \%$ fetal calf serum and 2 $\mathrm{mM}$ sodium pyruvate, $\mathrm{pH}$ 7.4]. Prior to the measurements, the microplates were incubated in a $\mathrm{CO}_{2}$-free incubator at $37^{\circ} \mathrm{C}$ for $1 \mathrm{~h}$. Oligomycin (Seahorse Bioscience), carbonyl cyanide 4-(trifluoromethoxy) phenylhydrazone (FCCP) (Seahorse Bioscience), and a mixture of rotenone and antimycin A (Seahorse Bioscience) were used for OCR analysis. The compounds were serially injected into the cells to measure mitochondrial respiration.

\section{ATP measurement}

Total cellular ATP content was determined using a luminescent ATP detection kit (PerkinElmer Life Sciences, Boston, MA, USA), according to the manufacturer's instructions. The luminescence intensity was measured with a Victor 3 luminometer (PerkinElmer). 


\section{Nude mouse xenograft experiment}

Six-week-old BALB/c nude mice were purchased from the Laboratory Animal Center of Southern Medical University and housed in the pathogen-free mouse facility of Guangzhou Medical University. All procedures for animal experiments were approved by the Ethics Committee of the Third Affiliated Hospital of Guangzhou Medical University (GD2019-022). Mice were subcutaneously injected with PBS suspensions of KBM5, KBM5-shDRP1, or KBM5-DRP1-OE cells at a density of $1 \times 10^{8}$ cells $/ \mathrm{mL}$. Ten days after injection, mice were randomly divided into eight groups (KBM5, KBM5+IM, KBM5-shDRP1+IM, KBM5-DRP1-OE+IM, KBM5-STI, KBM5-STI+IM, KBM5-STI+Mdivi-1, or KBM5-STI+IM+Mdivi-1). Mice were administered with either IM $(100 \mathrm{mg} / \mathrm{kg} / \mathrm{d})$ and/or Mdivi-1 $(30 \mathrm{mg} / \mathrm{kg} / \mathrm{d})$ daily when the tumor volume reached nearly $200 \mathrm{~mm}^{3}$. Tumor volumes were measured every 3 days for 25 days. The mice were sacrificed and the tumors were harvested 25 days later.

\section{Statistical analysis}

All experiments were repeated at least three times. Data represent mean \pm SEM. GraphPad Prism 5 (GraphPad Software Inc., San Diego, CA, USA) was used for all statistical analyses, and P > 0.05 was considered significant. Comparisons between two groups were performed using Student's t-test (twotailed) and comparisons between multiple groups were performed by one-way ANOVA. Survival analysis was performed using the Kaplan-Meier method.

\section{Results}

\section{Differentially expressed genes identified between imatinib-treatment responder and non-responder patient samples}

RNA samples from peripheral blood mononuclear cells were obtained from seven patients with CML [20]. Of these, three were classified in the R group and four in the NR group. After screening, there were 1013 differentially expressed genes, of which 488 were upregulated and 525 were downregulated in the NR group (Figure 1a). Furthermore, a heatmap was used to cluster differentially expressed genes (Figure 1b). Visually, the differentially expressed genes from the R and NR groups showed good clustering effects.

To investigate the roles of differentially expressed genes in imatinib response in CML, we used GO and KEGG pathway enrichment analysis. The biological process (BP) subcategory was mainly involved in the apoptotic process, metabolic process, and regulation of phosphorylation (Figure 1c). For the analysis of molecular function (MF), the elemental activities of the genes produced at the molecular level, the most significantly differentially expressed proteins were enriched in catalytic activity, metabolite binding, and metabolic enzyme activity (Figure 1d). Moreover, the cellular component (CC) analysis demonstrated that most of the differentially expressed proteins were located in the cytoplasm, cell membrane, and intrinsic component of the membrane (Figure 1e). Interestingly, these data from the $\mathrm{GO}$ analysis indicated a close relationship between mitochondria and differentially expressed proteins. In line with this, the pathways 
tuberculosis, and pathways in cancer (Figure 1f). Similarly, most of these pathways correlated well with mitochondrial function.

\section{Imatinib-resistant CML cells show higher active expression of DRP1 and a distinct mitochondrial morphology}

We previously utilized bioinformatics analyses to identify genes whose production was unique to imatinib-resistant CML cells. These data showed that the mitochondria were vital to the drug resistance of CML cells and could be a potential drug target. Of note, the mitochondria play a central role in cellular life and death decisions, and recent research points out a strong interplay between mitochondrial dynamics and cancer [21]. Given the role of mitochondrial dynamics in cancer, we next examined mitochondrial morphology in KBM5 cells in comparison with KBM5-STI cells using confocal and transmission electron microscopy (TEM). For confocal microscopy experiments, we labeled the mitochondria and nuclei of KBM5 cells and KBM5-STI cells with the mitochondrial dye Mito-Tracker and the DNA dye DAPI, respectively. Compared with KBM5 cells, KBM5-STI cells demonstrated a significantly lower mitochondrial-to-nuclear area ratio, suggesting that they have less mitochondrial content (Figure $2 \mathrm{a}$ and $2 b$ ). Consistently, the TEM images showed a lower average mitochondrial length (a typical indicator of mitochondrial fission) in KBM5-STI cells than in KBM5 cells (Figure 2c). In western blot analysis, a larger expression of mitochondrial fission protein DRP1 in mitochondrial content was observed in KBM5STI cells than KBM5 cells. While no change in DRP1 expression in the cytoplasm was detected between these two CML cell lines, the relative activity of DRP1 was significantly higher in KBM5-STI cells, further confirming their increased mitochondrial fission (Figure 2d).

To investigate the relationship between DRP1 expression and mitochondrial morphology in CML cells, the mitochondrial morphology of KBM5 cells after different treatments was assessed. In TEM images, mitochondrial elements became noticeably (although not significantly) elongated and interconnected in KBM5 cells with DRP1 knockdown or Mdivi-1 treatment when compared with those in control cells (Figure 2c). In contrast, the percentage of fragmented mitochondria was remarkably increased in KBM5 cells overexpressing DRP1 and in KBM5-STI cells (Figure 2c). All these results indicated that DRP1-mediated mitochondrial fission could take part in maintaining imatinib resistance in CML cells.

\section{Blockade of DRP1 disrupts mitochondrial respiration in CML}

In cancer cells, mitochondrial oxidative metabolism is crucial for energy production; therefore, blocking mitochondrial functions may have a therapeutic benefit [22]. In cultured KBM5 cells, mitochondrial oxygen consumption analyses showed that cells exhibited a higher OCR after imatinib stimulation compared to their counterparts [23]. By contrast, suppression of DRP1 had an opposite effect (in terms of OCR curve, basal and maximal OCR values, and ATP content) in KBM5 cells upon treatment with Mdivi-1, even in the combination group (Figure 3a-d). Experiments on KBM5-STI cells indicated that both imatinib and Mdivi-1, either alone or in combination, had similar results in KBM5 cells (Figure 3e-h). However, imatinib stimulation did not alter the OCR value and ATP content of KBM5-shDRP1 cells (Figure 3i-I).

Loading [MathJax]/jax/output/CommonHTML/fonts/TeX/fontdata.js pressing (DRP1-OE) cells had similar OCR 
values and ATP content when compared with KBM5 cells subjected to imatinib treatment (Figure 3i-I). Accordingly, a decrease in oxygen consumption upon DRP1 inhibition demonstrated that oxygen consumption was tightly linked to DRP1 in these cells.

Although the abovementioned results revealed that DRP1 is essential for mitochondrial respiration in $\mathrm{CML}$ cells, the related molecular mechanism is unknown. Cyclin-dependent kinase 5 (CDK5) and calcium/calmodulin-dependent protein kinase type 2 (CAMK2) have been reported to regulate the activity of DRP1 [23]. Targeting DRP1 expression by shRNA in KBM5 cells resulted in lower CDK5 and higher CAMK2 expression (Figure 3m-n). Conversely, in KBM5-DRP1-OE cells and KBM5-STI cells, there was an opposite change in CDK5 and CAMK2 expression (Figure $3 \mathrm{~m}-\mathrm{n}$ ). These results suggested that CDK5 and CAMK2 may be upstream drivers of DRP1 in CML cells by activating and inhibiting DRP1, respectively. AMP-activated protein kinase (AMPK) is a central cellular sensor of energy stress that plays a key role in determining the survival of cells under metabolic stress. We observed that knockdown of DPR1 promoted AMPK activation. Enhanced DRP1 expression (either in KBM5-DRP1-OE cells or KBM5-STI cells) decreased AMPK activation. These results suggest that DRP1 serves as an important node in response to metabolic stress in CML cells by AMPK regulation.

\section{Inhibiting DRP1 promotes CML mitochondria-dependent apoptosis}

To explore the detailed mechanism underlying the role of DRP1 in cell survival, we first assessed the effect of DRP1 in the apoptosis of CML cells with different levels of DRP1 expression. After imatinib treatment, the percentages of total (both early and late) apoptotic KBM5 cells were significantly higher under both DRP1 knockdown and Mdivi-1 treatment when compared with control cells (Figure 4a). In contrast, KBM5 cells overexpressing DRP1 and KBM5-STI cells were resistant to imatinib-induced apoptosis (Figure 4a). Additionally, apoptosis of cells with high DRP1 expression (both in the KBM5DRP1-OE cells and KBM5-STI cells) was significantly increased by the combination of imatinib with Mdivi-1, suggesting that inhibition of DRP1 enhanced imatinib-induced apoptosis of CML cells, even in KBM5-STI cells (Figure 4a).

When apoptosis is initiated, the pro-apoptotic protein cytochrome $\mathrm{c}$ is released from the mitochondrial inner membrane into the cytoplasm to activate caspase-9 and trigger the apoptotic cascade. Western blotting results showed that the levels of cytochrome c, caspase-9, and caspase- 3 were significantly increased after Mdivi-1 treatment, compared with the untreated cells KBM5 cells and KBM5-STI cells (Figure 4b). This confirmed the pro-apoptotic role of DRP1 inhibition in CML cells.

\section{DRP1 depletion is accompanied with inhibition of autophagy}

Many studies have revealed that autophagy can protect cells by preventing apoptosis, especially in cancer cells [24]. We then examined whether autophagy plays a key role in DRP1-mediated inhibition of CML cell apoptosis. In Figure 5a and 5b, imatinib treatment significantly increased the conversion of the cellular protein LC3-I to LC3-II (which are markers of autophagy) and decreased the expression of 


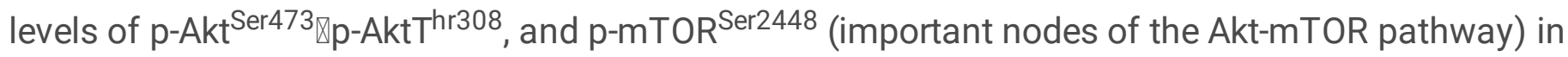
KBM5 cells and KBM5-STI cells. In contrast, DRP1 downregulation with Mdivi-1 reversed these changes, even in the combination treatment groups from both cell lines (Figure $5 a$ and $5 b$ ). In order to test the effect of DRP1 expression on autophagy in CML cells, we next performed shRNA-mediated knockdown and overexpression studies in KBM5 cells. We observed that DRP1-depleted KBM5 cells had a significantly decreased LC3- $\mathbb{Z} / \mathbb{Q}$ ratio and an increased expression of p62 and activity of the Akt-mTOR pathway after imatinib treatment, relative to KBM5 cells under similar conditions (Figure 5c). Interestingly, we also found that there was no change in autophagy activity between KBM5 cells with DRP1 high expression and their counterparts upon imatinib stimulation (Figure $5 \mathrm{c}$ ). These data suggest that DRP1depleted CML cells are resistant to imatinib-related autophagy induction.

To further test this hypothesis, we examined KBM5 cells and KBM5-STI cells with different DRP1 expression using a novel permeable and LC3-special incorporated fluorescent probe for monitoring autophagy by confocal fluorescence microscopy. In the confocal fluorescence images, there was an appreciable increase in green fluorescent spots distributed throughout the cytoplasm or accumulated in the perinuclear region of KBM5 cells and KBM5-STI cells after treatment with imatinib (Figure $5 \mathrm{~d}$ and $5 \mathrm{e}$ ). However, in both cell lines, Mdivi-1 treatment did not show an obvious green fluorescence when compared with the control cells (Figure $5 \mathrm{~d}$ and $5 \mathrm{e}$ ). In the combination treatment groups of both cell lines, pharmaceutical inhibition of DRP1 with Mdivi-1 significantly suppressed green fluorescence expression relative to the imatinib-treatment groups (Figure $5 d$ and $5 e$ ). These changes were also observed in KBM5 cells with a genetic inhibition of DRP1 after imatinib stimulation. In contrast, imatinib markedly increased the quantity and intensity of green fluorescence in KBM5 cells overexpressing DRP1 when compared with KBM5-shDRP1 cells (Figure 5f). Conversely, KBM5-DRP1-OE cells had comparable LC3-fluorescence expression with that of their counterparts after imatinib treatment (Figure 5f). Collectively, our results indicated that inhibition of DRP1 blocked the activation of autophagy in CML cells under imatinib treatment.

\section{DRP1 inhibition enhances imatinib sensitivity of CML cells in vivo}

To confirm the possible role of DRP1 inhibition in reversing the drug resistance of CML cells, we examined the effect of pharmacological or genetic inhibition of DRP1 on tumor growth in vivo by constructing a xenograft nude mouse model using KBM5 cells with different DRP1 expression or KBM5STI cells. We transplanted KBM5, KBM5-shDRP1, KBM5-DRP1-OE, or KBM5-STI cells into recipient immunocompromised mice, allowed leukemia to develop, and at that point administered the mice with imatinib, Mdivi-1, or a combination treatment. Indeed, in mice with KBM5 cells, imatinib treatment resulted in a significant reduction in tumor volume and weight relative to the untreated group (Figure 6ac). Next, we tested the effect of altered mitochondrial fission on tumor growth in vivo by constructing a mouse CML model using KBM5 cell lines with stable DRP1 knockdown or overexpression. As shown in Figure 6a-c, the size and weight of the tumor mass in mice bearing KBM5 cells with DRP1 low expression were decreased by imatinib treatment when compared with the ones with KBM5 cells, while DRP1 
DRP1 inhibition and imatinib on CML cells harboring the T315I mutation. Our results showed that there were no significant changes in the sizes and weights of subcutaneous tumors after imatinib treatment when compared with control tumors, whereas the tumor size and weight of xenografts developed from KBM5-STI cells after mvidi-1 treatment exhibited a significant decrease compared with controls (Figure $6 \mathrm{~d}-\mathrm{f})$. Notably, the combination treatment of Mdivi-1 and imatinib remarkably inhibited the growth capacity of tumors induced by KBM5-STI cells (Figure 6d-f). These results suggested an additive effect of DRP1 inhibition on human CML.

\section{Discussion}

Despite the remarkable improvements in the treatment of CML patients since the introduction of TKIs, which induced long-term overall survival rates higher than $90 \%$, the existence of TKI resistance and side effects still result in treatment failure, highlighting the need for novel treatment options [25]. Notably, point mutations in the BCR-ABL kinase domain are the most frequent resistance mechanisms after TKI treatment [4]. However, recent novel biological evidence has showed that not only BCR-ABL-dependent, but also BCR-ABL-independent mechanisms are involved in TKI treatment resistance [5-9].

In the 1920s, Otto Warburg and colleagues demonstrated that cancer cells display increased glycolysis under aerobic conditions [26]. Since then, the importance of mitochondrial function has been undervalued in cancer. However, at the end of the last millennium, several pioneering studies have brought mitochondria back into the focus of different research fields. These studies put forward the mitochondrion as a central hub in cell biology by providing evidence of its key role in signal transduction, cell death regulation, stemness maintenance, as well as bioenergy production and biosynthesis $[27,28]$. Similar to embryonic stem cells and induced pluripotent stem cells, cancer stem cells are characterized by de-differentiation and maintenance of stemness which contain perinuclear-localized fragmented mitochondria by remodeling of mitochondrial functions[29]. Moreover, mitochondrial morphology that reflects mitochondrial fission and fusion is also particularly important for its functions [30]. However, the biological significance of mitochondrial dynamics in cancer drug resistance remains largely unknown. In this study, we used bioinformatic analyses and a confocal microscopy experimental approach to reveal that imatinib-resistant CML cells (including CML cells with T315I mutation) develop a dependency on mitochondrial functions, with notably fragmented mitochondria compared with their imatinib-sensitive counterparts. Although the expression of DRP1 in the cytoplasm did not differ between KBM5-STI cells (CML imatinib-resistant cells carrying BCR-ABL ${ }^{T 3151}$ ) and KBM5 (imatinib-sensitive cells), KBM5-STI cells exhibited a strikingly higher expression of DRP1 in mitochondria, where it is activated. The ectopic expression of a gain-of-function DRP1 construct in KBM5 cells, which led to a hyper-activation of DRP1, promoted mitochondrial fragmentation. Upon inhibition of DRP1, more diffuse mitochondria were observed in KBM5 cells, suggesting that DRP1 is required to sustain the unique mitochondrial morphology in CML cells.

Notably, mitochondrial fission is important for the segregation of dysfunctional mitochondria, and an

Loading [MathJax]/jax/output/CommonHTML/fonts/TeX/fontdata.js legradation of dysfunctional mitochondria and

Page 10/21 
an accumulation of mitochondrial stress damage [31]. PINK1 and Parkin, mitophagy related regulators, participate in the downregulation of mitochondrial fusion by integrating mitofusin 1 and mitofusin 2 [32,33]. Moreover, BNIP3 induces DRP1 mitochondrial translocation to promote mitochondrial fission [34]. In our data, we also demonstrated that the inhibition of DRP1 by the mitochondrial fission inhibitor Mdivi1 remarkably decreased imatinib-related autophagy by activating PI3K-Akt-mTOR signaling. Meanwhile, inhibition of autophagy by Mdivi-1 re-sensitized resistant CML cells (including CML cells harboring the T315I mutation) to imatinib treatment. This probably occurs because inhibition of mitochondrial fission leads to a blockade of autophagy and promotes cell apoptosis.

Therefore, it is not surprising that the combination treatment of imatinib and DRP1 inhibition produced synergistic anti-leukemic effects in CML cells, including cells carrying the T315I mutation. DRP1 inhibition has recently emerged as a promising therapeutic approach in various tumor types, including pancreatic adenocarcinoma, nasopharyngeal carcinoma, glioblastoma, lung adenocarcinoma, breast cancer, and melanoma cells [35]. Importantly, Xie and colleagues demonstrated that gene silencing of DRP1 impaired the proliferation of cancer stem cells, but not glioblastoma non-cancer stem cells or neural progenitor cells [15]. It seems that DRP1 inhibition may be a safe cancer-specific therapy. Mdivi-1 is a selective inhibitor of DRP1 that acts by blocking the self-assembly of DRP1 and impairing its GTPase activity, according to a previous study [36]. However, recent research has indicated that Mdivi-1 influences the electron transport chain, which might result in off-target effects [37]. Another novel DRP1 inhibitor, P110, displays selective DRP1 inhibition, which avoids off-target effects, as observed in a neurodegenerative study [38]. Therefore, several proof-of-concept experiments are still needed to improve the understanding of DRP1 in cancer drug resistance, expedite the search for safe and effective DRP1targeting molecules, and support the potential clinical use of such therapies.

An oxidative phosphorylation (OXPHOS)-dependent metabolic status has been found to be responsible for resistance to oncogene inhibition, radiation, and chemotherapeutics in many types of cancers [39]. Recent work by Kuntz and colleagues demonstrated that CML stem cells rely on mitochondrial OXPHOS, and that combined imatinib and the mitochondrial protein translation inhibitor tigecycline could effectively eradicate CML stem cells [40].Our previous work reached a similar conclusion in CML cells with the T315I mutation [23]. Upon imatinib treatment, both CML-sensitive and CML-resistant cells had increased levels of mitochondrial OXPHOS. Blockage of DRP1 contributed to a downregulation of imatinib-related mitochondrial OXPHOS promotion by activating downstream AMPK signaling. AMPK serves as a central cellular sensor of energy stress, suggesting a key function in determining the survival of cells suffering from metabolic stress [42]. A recent study indicated that DRP1 acts as a critical node in the response of brain tumor-initiating cells to metabolic stress though AMPK regulation in glioblastomas [43]. Thus, we believe that DRP1-mediated mitochondrial fission participates in cancer resistance by disturbing mitochondrial OXPHOS, suggesting that DRP1 inhibition could have potential applications for the clinical improvement of patients suffering from different types of cancers.

\section{Conclusions}

Loading [MathJax]/jax/output/CommonHTML/fonts/TeX/fontdata.js

Page $11 / 21$ 
In summary, our study demonstrated that imatinib-resistant CML cells display different gene profiles that focus on mitochondrial function compared to their drug-sensitive counterparts. Resistant CML cells showed distinct fragmented mitochondria and an overexpression of DRP1. Furthermore, increased levels of DRP1 were associated with a lower apoptotic rate, higher autophagy levels, and stronger mitochondrial respiration. Targeted inhibition of DRP1 using both DRP1 chemical inhibition and gene silencing resulted in increased cell death, decreased autophagy, and a downregulation of mitochondrial respiration in CML cells. Combined imatinib and DRP1 inhibition produced synergistic anti-leukemic effects in CML cells, including KBM5-STI cells. We also extended these findings to a mouse model harboring KBM5 or KBMBSTI cells in vivo and completed secondary transplantation and survival analysis in the future.

Mechanically, we reported that CML cells exhibiting BCR-ABL T315I remain in the mitochondrial fission state, mediated by CDK5 /CAMK2-DRP1-AMPK axis. This model suggests that inhibition of DRP1-related combination treatment may represent a promising strategy to eradicate CML cells, including that of treatment-resistant $\mathrm{CML}$ cells harboring the T315I mutation.

\section{Declarations}

\section{Acknowledgements}

We are very grateful to Prof. Michael Andreeff and Prof. Hongsheng Zhou for the KBM5-STI cells. We would like to express our sincere gratitude to Tong Lin. ZL thanks Qilong Liang for his continuous care and endless love. I want to grow old with you.

\section{Funding}

This work was supported by the Natural Scientific Fund of Guangdong Province (2018A030310299), the Science and Innovation Strategy Fund of Guangdong Province (2019A1515010390) and the Elite Project Fund of The Third Affiliate Hospital of Guangzhou Medical University (2018001).

\section{Availability of data and materials}

All data generated or analyzed during this study are included in this published article. Further details are available on request.

\section{Author's contributions}

$Z L$ and $Z W$ designed the experiments, performed the experiments, analyzed the data, and wrote the manuscript. JT,YL,GL,XC, BL, and QQ contributed to the experimental work. All authors have read and approved the final manuscript.

\section{Competing interests}

The authors declare that they have no competing interests. 
Not applicable.

\section{Ethics approval and consent to participate}

This study was approved by the Medical Ethics Committee of the Third Affiliated Hospital of Guangzhou Medical University(GD2019-022) and performed in accordance with the Declaration of Helsinki.

\section{References}

1. Baccarani M, Deininger MW, Rosti G, et al. European LeukemiaNet recommendations for the management of chronic myeloid leukemia: 2013. Blood. 2013; 122(6):872-84.

2. Garcia-Gutierrez V, Hernandez-Boluda JC. Tyrosine kinase inhibitors available for chronic myeloid leukemia: efficacy and safety. Front Oncol. 2019; 9:603.

3. Soverini S, Mancini M, Bavaro L, et al. Chronic myeloid leukemia: the paradigm of targeting oncogenic tyrosine kinase signaling and counteracting resistance for successful cancer therapy. Mol Cancer. 2018;17(1):49.

4. Soverini S, Branford S, Nicolini FE, et al. Implications of BCR-ABL1 kinase domain-mediated resistance in chronic myeloid leukemia. Leuk Res. 2014;38(1):10-20.

5. Mahon FX, Belloc F, Lagarde V, et al. MDR1 gene overexpression confers resistance to imatinib mesylate in leukemia cell line models. Blood. 2003;101(6):2368-73.

6. Illmer T, Schainh M, Platzbecker $U$, et al. P-glycoprotein-mediated drug efflux is a resistance mechanism of chronic myelogenous leukemia cells to treatment with imatinib mesylate. Leukemia. 2004;18(3): 401-8.

7. White DL, Saunders VA, Dang P, et al. Most CML patients who have a suboptimal response to imatinib have low OCT-1 activity: higher doses of imatinib may overcome the negative impact of low OCT-1 activity. Blood. 2007;110(12): 4064-72.

8. Giustacchini A, Thongjuea S, Barkas N, et al. Single-cell transcriptomics uncovers distinct molecular signatures of stem cells in chronic myeloid leukemia. Nat Med. 2017;23(6):692-702.

9. Mendez-Ferrer S, Michurina TV, Ferraro F, et al. Mesenchymal and haematopoietic stem cells form a unique bone marrow niche. Nature. 2010;466(7308): 829-34.

10. Soverini S, Hochhaus A, Nicolini FE, et al. BCR-ABL kinase domain mutation analysis in chronic myeloid leukemia patients treated with tyrosine kinase inhibitors: recommendations from an expert panel on behalf of European LeukemiaNet. Blood. 2011;118(5): 1208-15.

11. Cortes JE, Kim DW, Pinilla-lbarz, J, et al. Ponatinib efficacy and safety in Philadelphia chromosomepositive leukemia: final 5-year results of the phase 2 PACE trial. Blood. 2018;132(4):393-404.

12. Sharma A, Smith HJ, Yao P, et al. Causal roles of mitochondrial dynamics in longevity and healthy aging. EMBO Rep. 2019;20(12):e48395.

13. Pernas L, Scorrano L. Mito-Morphosis: mitochondrial fusion, fission, and cristae remodeling as key 6;78:505-31. 
14. Wallace, DC. Mitochondria and cancer. Nat Rev Cancer. 2012;12(10):685-98.

15. Xie Q, Wu Q. Horbinski C, et al. Mitochondrial control by DRP1 in brain tumor initiating cells. Nat Neurosci. 2015;18(4):501-10.

16. Rehman J, Zhang HJ, Toth PT, et al. Inhibition of mitochondrial fission prevents cell cycle progression in lung cancer. FASEB J. 2012;26(5):2175-86.

17. Dal Yontem, F, Kim SH, Ding, Z, et al. Mitochondrial dynamic alterations regulate melanoma cell progression. J Cell Biochem. 2018.

18. Zhang J, Zhang Y, Wu W, et al. Guanylate-binding protein 2 regulates Drp1-mediated mitochondrial fission to suppress breast cancer cell invasion. Cell Death Dis. 2017;8(10): e3151.

19. Peiris-Pages M, Bonuccellim G, Sotgia F, et al. Mitochondrial fission as a driver of stemness in tumor cells: mDIVI1 inhibits mitochondrial function, cell migration and cancer stem cell (CSC) signalling. Oncotarget 2018;9(17): 13254-75.

20. Silveira RA, Fachel AA, Moreira YB, et al. Protein-coding genes and long noncoding RNAs are differentially expressed in dasatinib-treated chronic myeloid leukemia patients with resistance to imatinib. Hematology. 2014;19(1):31-41.

21. Altieri DC. Mitochondria on the move: emerging paradigms of organelle trafficking in tumour plasticity and metastasis. Br J Cancer. 2017;117(3):301-5.

22. Bosc C, Selak MA, Sarry JE. Resistance is futile: targeting mitochondrial energetics and metabolism to overcome drug resistance in cancer treatment. Cell Metab. 2017;26(5):705-707.

23. Lu Z, Xu N, He B, et al. Inhibition of autophagy enhances the selective anti-cancer activity of tigecycline to overcome drug resistance in the treatment of chronic myeloid leukemia. J Exp Clin Cancer Res. 2017;36(1):43.

24. Xie LL., Shi F, Tan Z, et al. Mitochondrial network structure homeostasis and cell death. Cancer Sci. 2018;109(12):3686-94.

25. Mulcahy, Levy JM, Thorburn A. Autophagy in cancer: moving from understanding mechanism to improving therapy responses in patients. Cell Death Differ. 2019.

26. Westerweel PE, Te, Boekhorst PAW, Levin, MD, et al. New approaches and treatment combinations for the management of chronic myeloid leukemia. Front Oncol. 2019;9:665.

27. Vander HM, Cantley LC, Thompson CB. Understanding the Warburg effect: the metabolic requirements of cell proliferation. Science. 2009;324(5930):1029-33.

28. Ott M, Gogvadze V, Orrenius S, et al. Mitochondria, oxidative stress and cell death. Apoptosis. 2007;12(5):913-22.

29. Wanet A, Arnould T, Najimi M, et al. Connecting mitochondria, metabolism, and stem cell fate. Stem Cells Dev. 2015;24(17):1957-71.

30. Chen $\mathrm{H}$, Chan DC. Mitochondrial dynamics in regulating the unique phenotypes of cancer and stem cells. Cell Metab. 2017;26(1):39-48. 
31. Fu W, Liu Y, Yin H. Mitochondrial dynamics: biogenesis, fission, fusion, and mitophagy in the regulation of stem cell behaviors. Stem Cells Int. 2019;9757201.

32. Otera, $\mathrm{H}$, Wang $\mathrm{C}$, Cleland MM, et al., Mff is an essential factor for mitochondrial recruitment of Drp1 during mitochondrial fission in mammalian cells. J Cell Biol. 2010;191(6):1141-58.

33. Ziviani E, Tao RN, Whitworth AJ. Drosophila parkin requires PINK1 for mitochondrial translocation and ubiquitinates mitofusin. Proc Natl Acad Sci USA. 2010;107(11):5018-23.

34. Chen Y, Dorn GN. PINK1-phosphorylated mitofusin 2 is a Parkin receptor for culling damaged mitochondria. Science. 2013;340(6131):471-5.

35. Lee Y, Lee HY, Hanna RA, et al. Mitochondrial autophagy by Bnip3 involves Drp1-mediated mitochondrial fission and recruitment of Parkin in cardiac myocytes. Am J Physiol Heart Circ Physiol. 2011;301(5):H1924-31.

36. Skoda J, Borankova K, Jansson PJ, et al. Pharmacological targeting of mitochondria in cancer stem cells: An ancient organelle at the crossroad of novel anti-cancer therapies. Pharmacol Res. 2019;139:298-313.

37. Cassidy-Stone A, Chipuk JE, Ingerman E, et al. Chemical inhibition of the mitochondrial division dynamin reveals its role in Bax/Bak-dependent mitochondrial outer membrane permeabilization. Dev Cell. 2008;14(2):193-204.

38. Bordt EA, Clerc P, Roelofs BA, et al. The putative Drp1 inhibitor mdivi-1 is a reversible mitochondrial complex I inhibitor that modulates reactive oxygen species. Dev Cell. 2017;40(6):583-594.e6.

39. Qi X, Qvit N, Su YC, et al. A novel Drp1 inhibitor diminishes aberrant mitochondrial fission and neurotoxicity. J Cell Sci. 2013;126(Pt 3):789-802.

40. Sica V, Bravo-San, Pedro JM, Stoll G., et al. Oxidative phosphorylation as a potential therapeutic target for cancer therapy. Int J Cancer. 2020;146(1):10-7.

41. Kuntz EM, Baquero P, Michie AM, et al. Targeting mitochondrial oxidative phosphorylation eradicates therapy-resistant chronic myeloid leukemia stem cells. Nat Med. 2017;23(10):1234-40.

42. Saito Y, Chapple RH, Lin A, et al. AMPK protects leukemia-initiating cells in myeloid leukemias from metabolic stress in the bone marrow. Cell Stem Cell. 2015;17(5):585-96.

43. Zhang S, Sheng $H$, Zhang $X$, et al. Cellular energy stress induces AMPK-mediated regulation of glioblastoma cell proliferation by PIKE-A phosphorylation. Cell Death Dis. 2019;10(3):222.

\section{Figures}


a

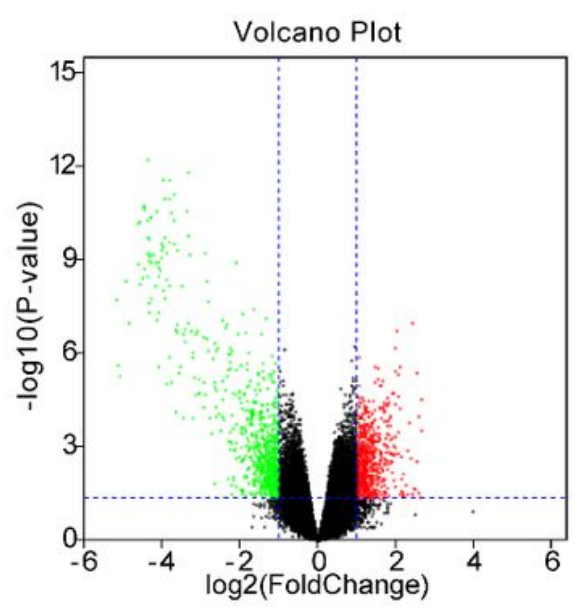

c

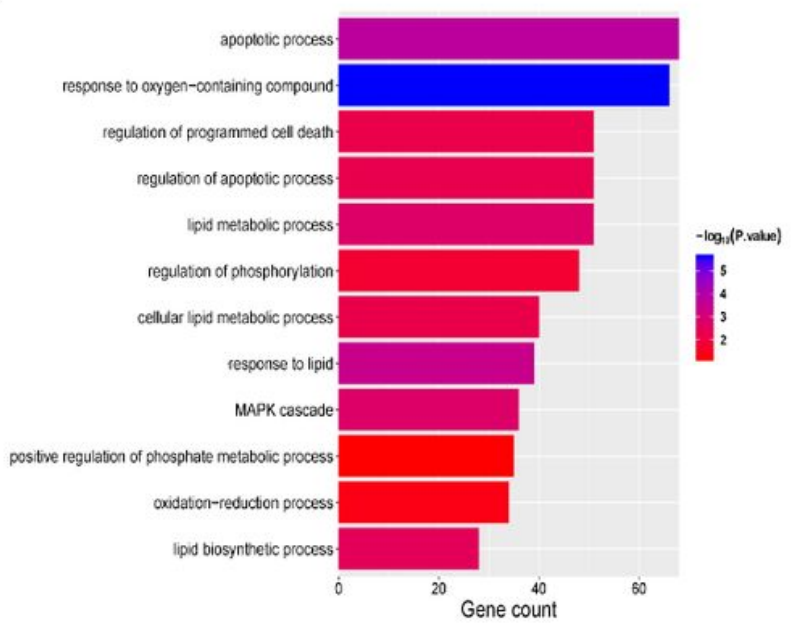

e

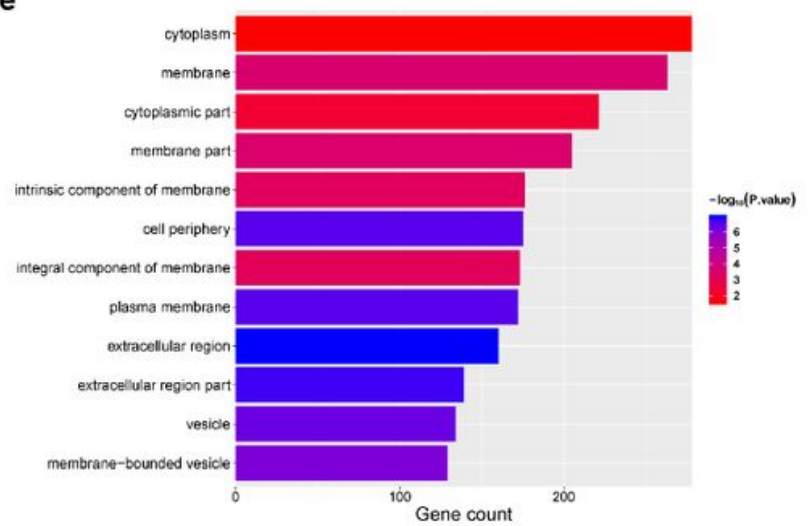

b

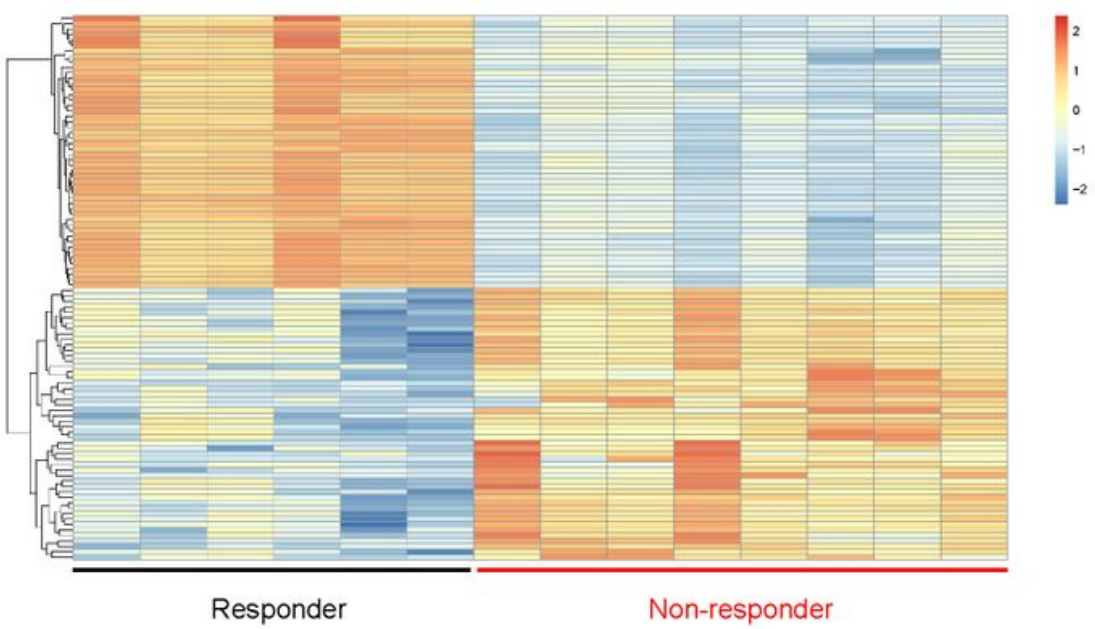

d

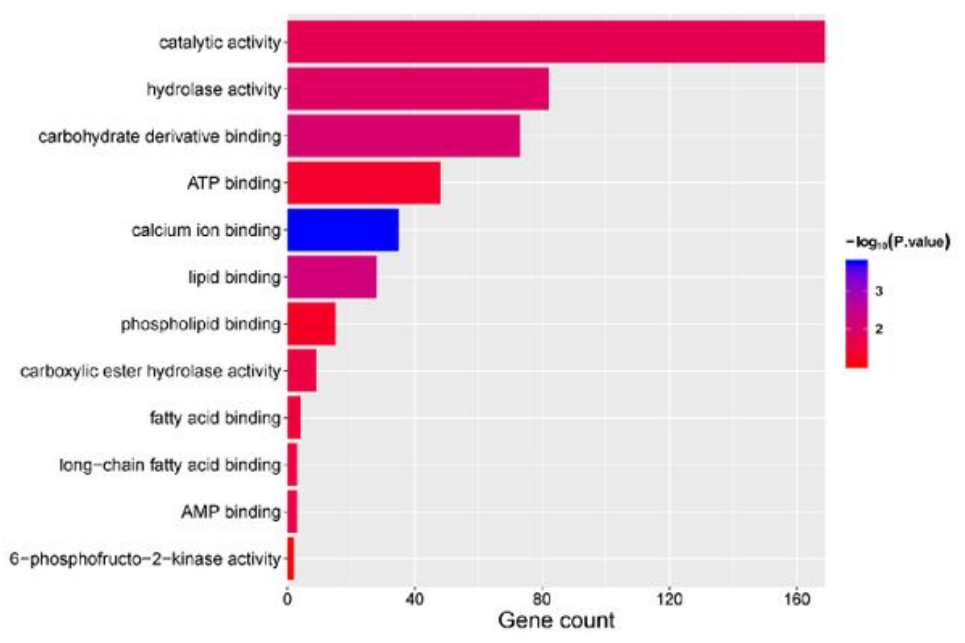

f

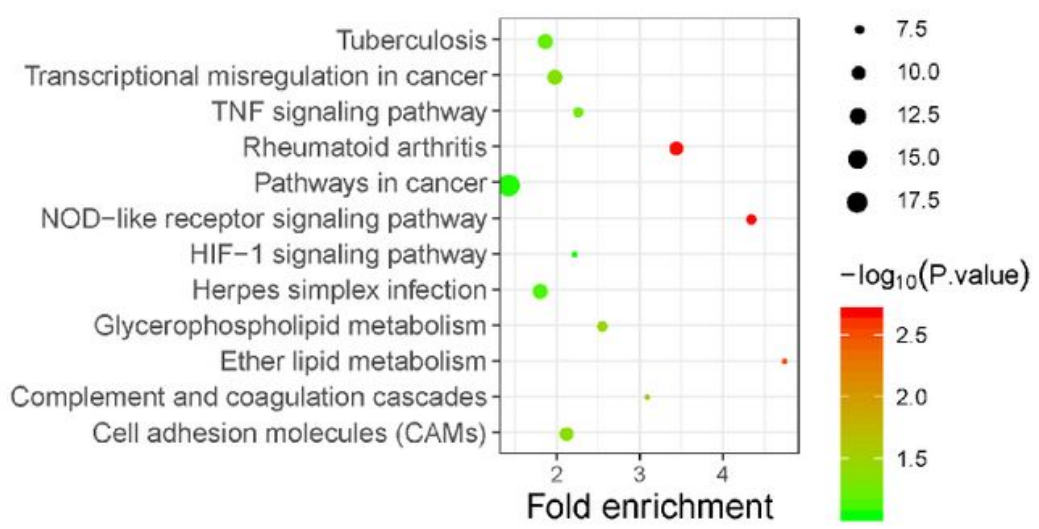

\section{Figure 1}

The differentially expressed genes between imatinib-treatment responder and non-responder patients analyzed through bioinformatics analysis. (a) The differentially expressed genes filtered through a volcano map. The identification criteria for differentially expressed genes were as follows: $p$-value $\mathbb{0} 0.01$ and fold change $₫ 2$ times were considered to be significantly different. (b) The hierarchical clustering of differentially expressed genes. The biological processes (c), molecular function (d), and cellular 
component (e) analysis of the differentially expressed genes. (f) The results of pathway analysis of the differentially expressed genes.

a
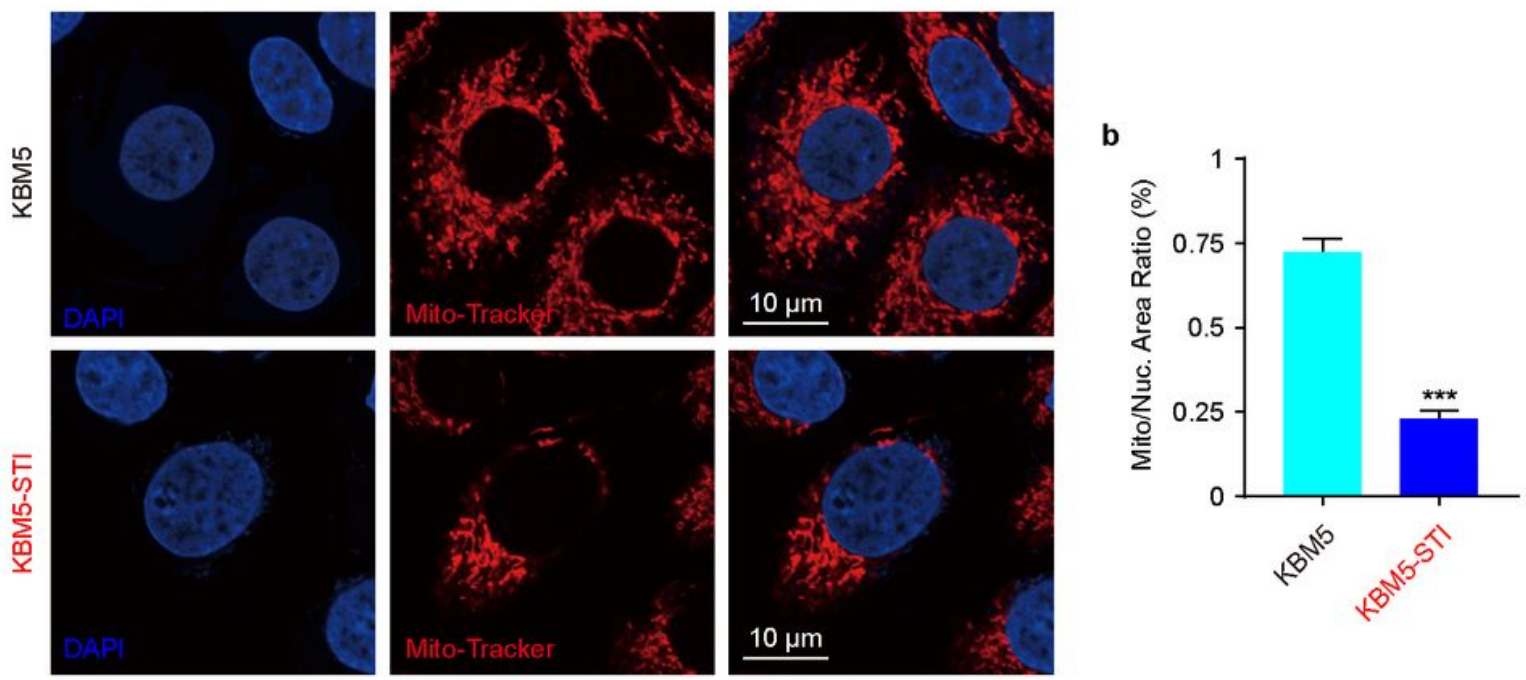

c

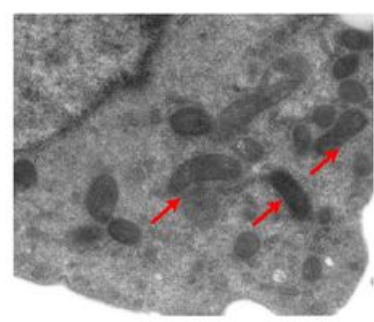

KBM5

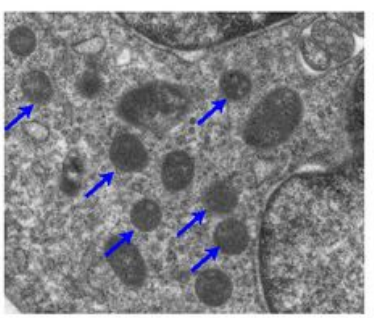

KBM5-STI

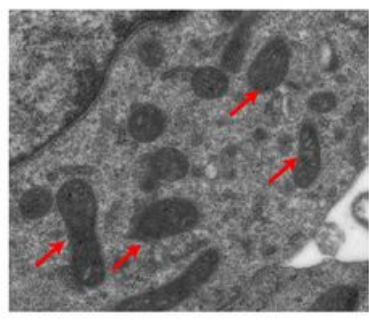

KBM5-shDRP1

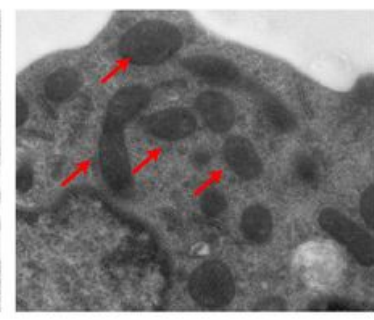

KBM5-Mdivi-1

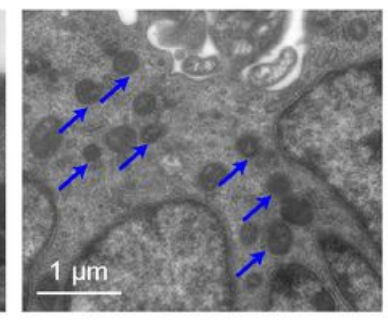

KBM5-DRP1-OE

d
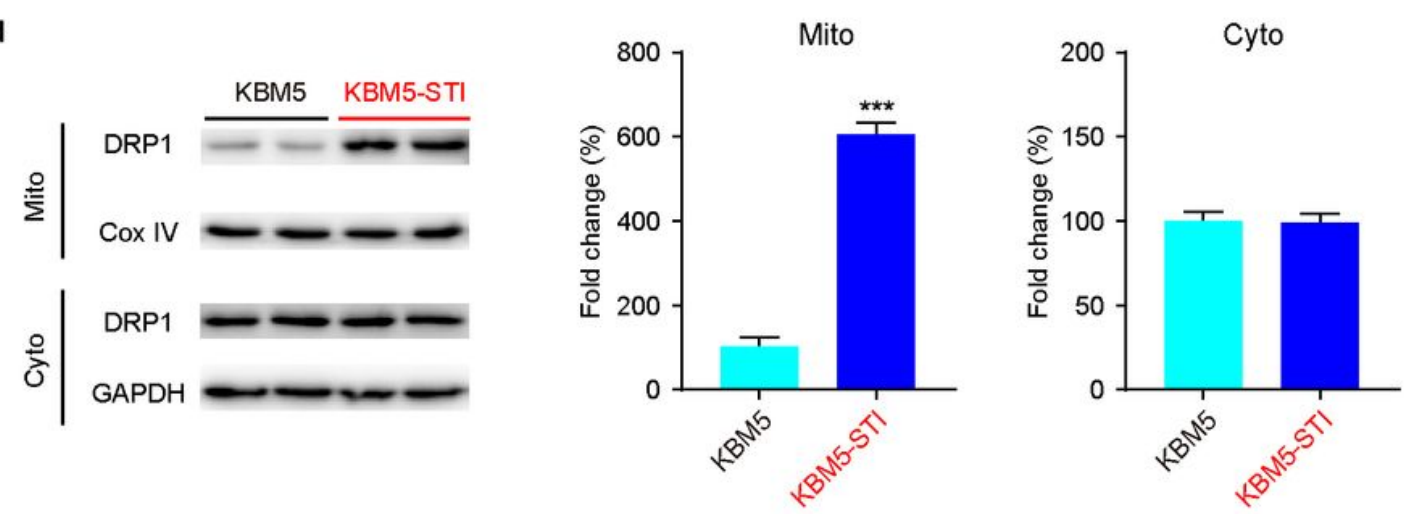

Figure 2

Imatinib-resistant CML cells have higher active expression of DRP1 and increased mitochondrial fission. (a) Representative confocal microscopy images showed the morphology of mitochondria in KBM5 cells versus KBM5-STI cells. (b) Mitochondrial-to-nuclear area ratio in KBM5 cells versus KBM5-STI cells. (c) Representative TEM images displayed the morphology of mitochondria in KBM5 cells with different treatment and KBM5-STI cells. Red and blue arrows highlight distinct mitochondrial morphology. (d) Western blotting results demonstrated expression of DRP1 protein in the cytoplasm and mitochondria in KBM5 and KBM5-STI cells, respectivelv. ${ }^{* *} \mathrm{D}<0.001$. 

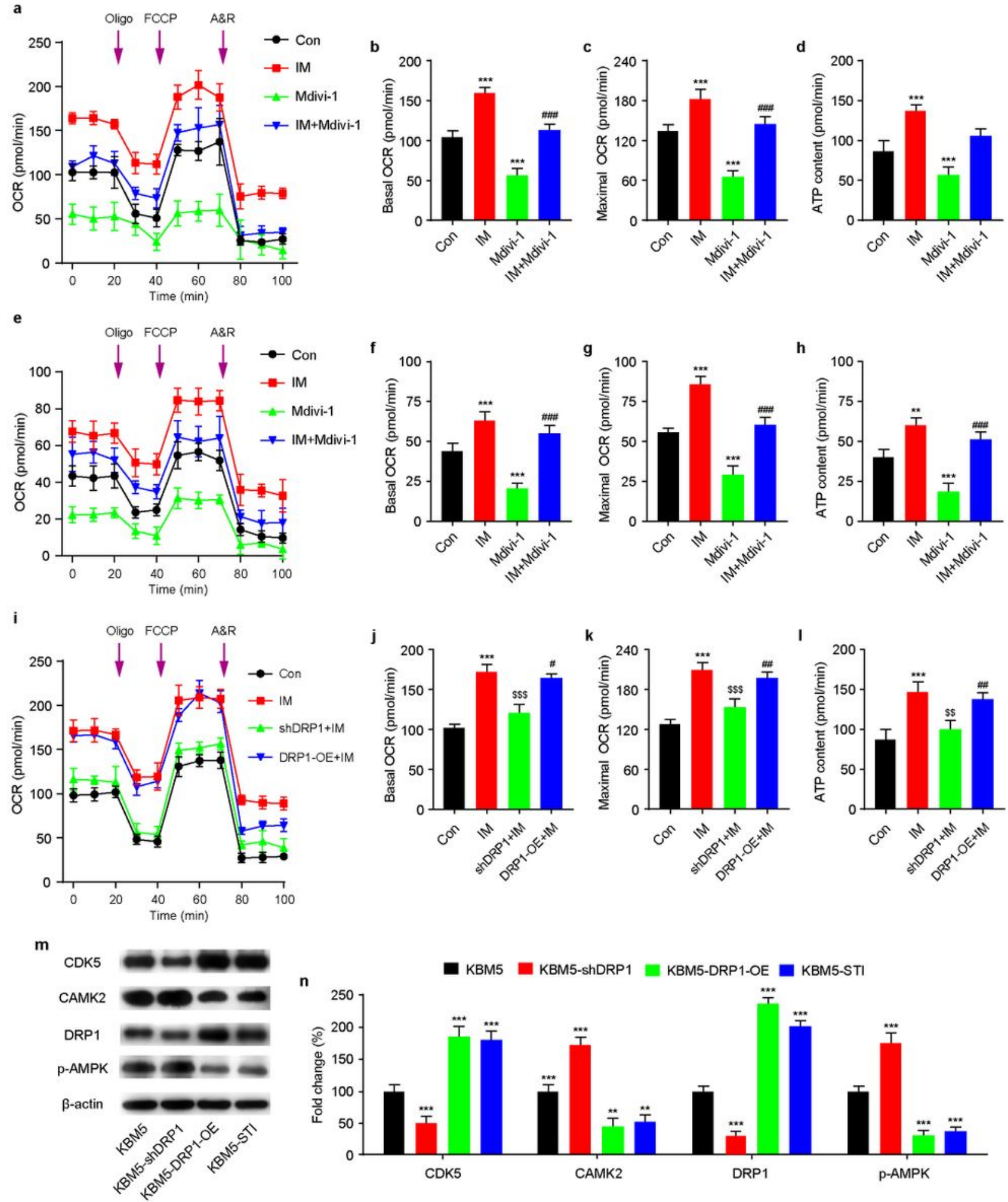

\section{Figure 3}

High-OXPHOS imatinib-resistant CML cells rely on the CDK5/CAMK2-DRP1-AMPK pathway. (a, e, i) Representative OCR pattern of KBM5, KBM5-STI, and KBM5 cells with different DRP1 expression after imatinib or/and Mdivi-1 treatment, respectively. (b, $f, j)$ Basal OCR were detected in these CML cell lines. (c, g, k) Maximal OCR were tested in these CML cell lines. ( $d, h, I)$ ATP content was measured in these 
CDK5/CAMK2-DRP1-AMPK pathway in CML cell lines with different DRP1 expression. Data were means of three replicates per cell line. ${ }^{*}, \#, \$$ indicate $p<0.05 . * \star \llbracket \# \#$,

$$
\text { indicate } \mathrm{p}<0.01 . * * * \# \# \# \text { \# }
$$

$\$$ indicate $p<0.001$.

a
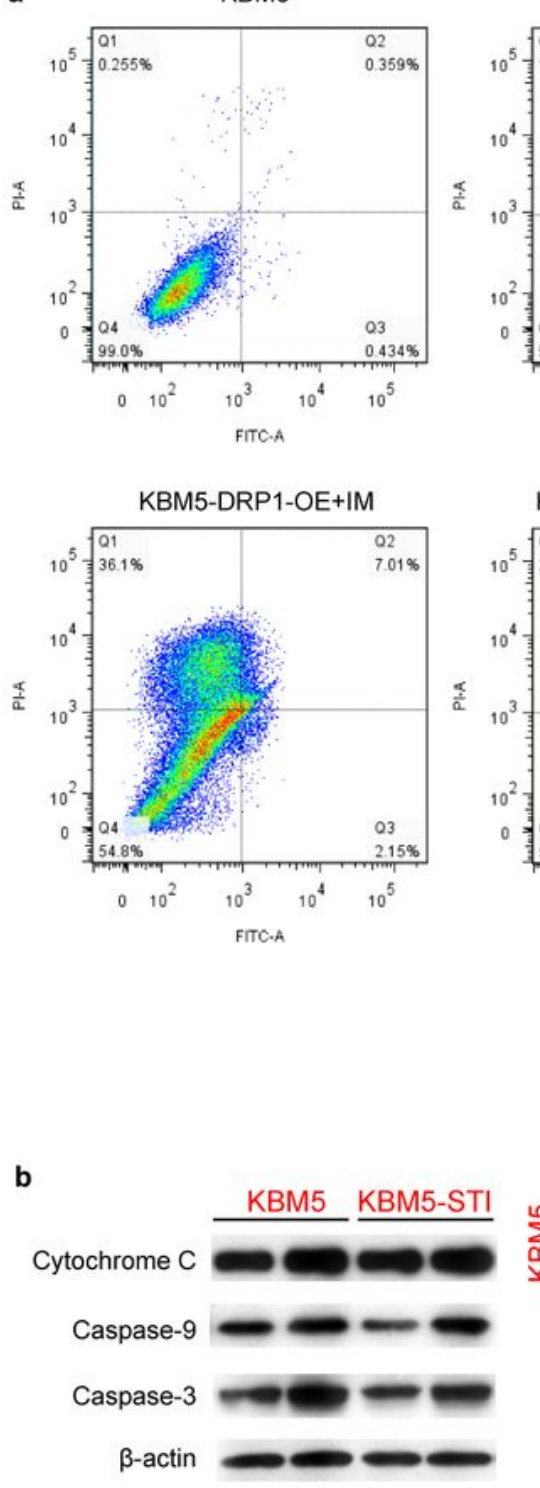

Mdivi-1 - $+\quad-+$
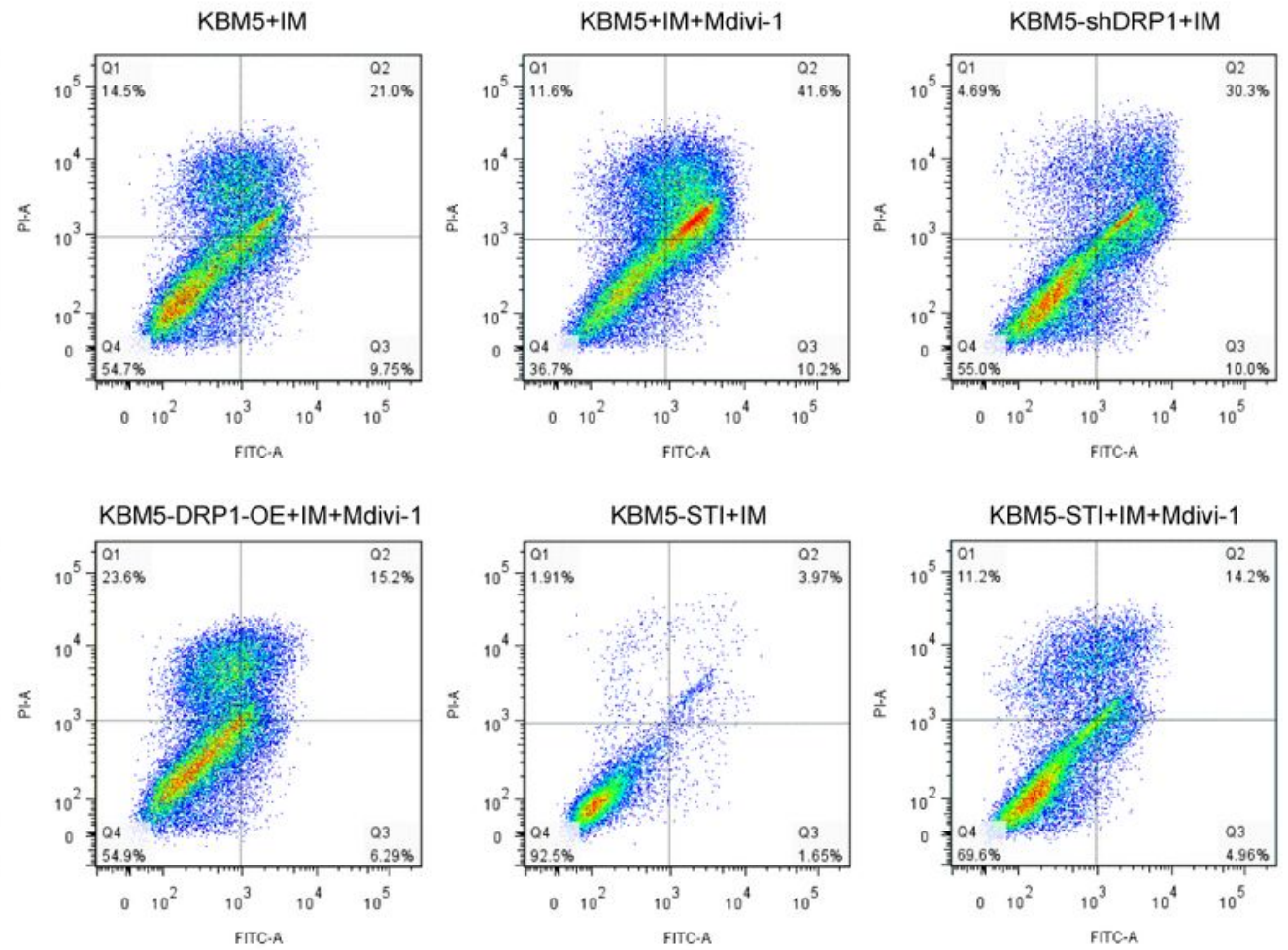

Cytochrome C
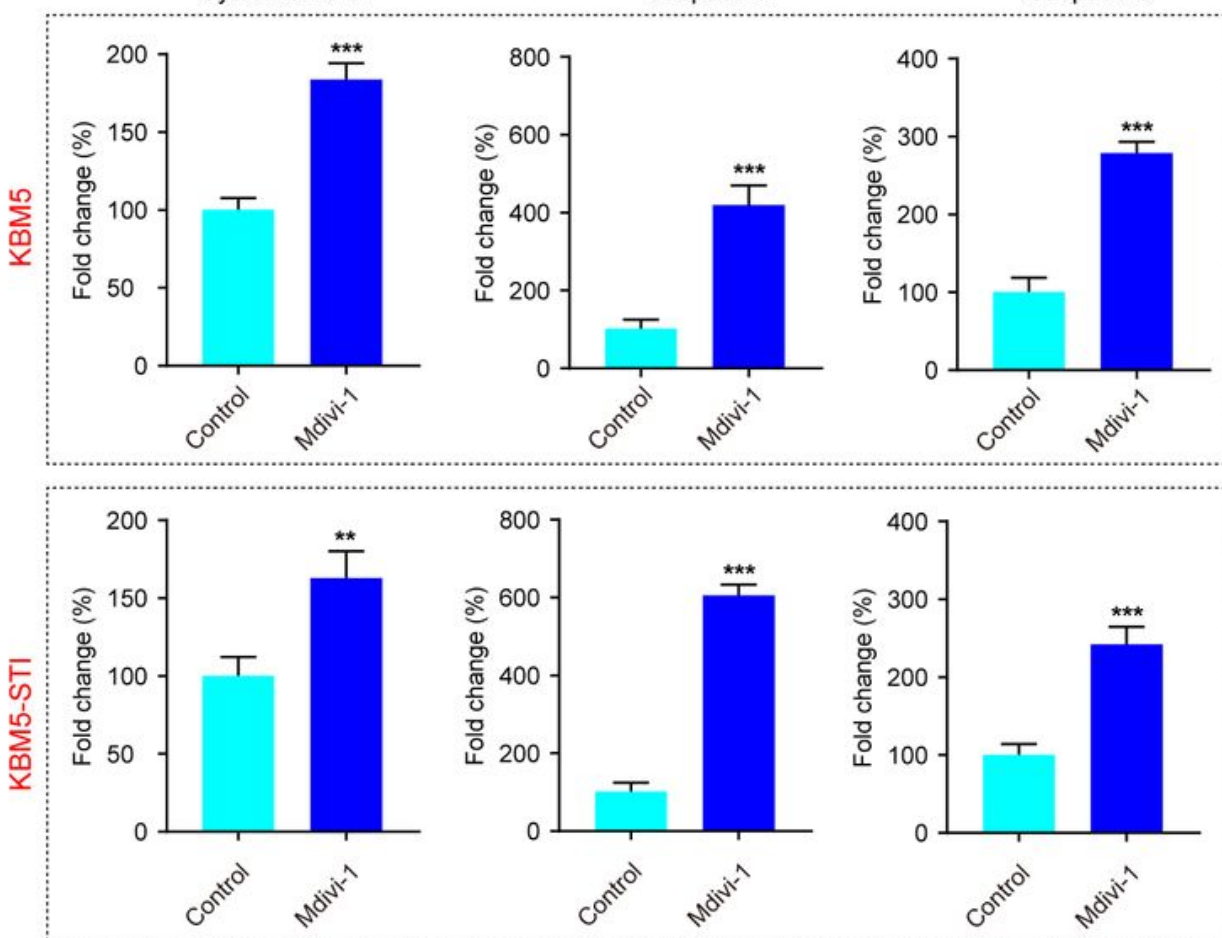

\section{Figure 4}


Inhibition of DRP1 induces apoptosis of CML cells by activating the cytochrome c/ capase-9/capase-3 pathway. (a) Apoptosis assay of the CML cells in response to treatment with DRP1 inhibition and/or imatinib. Apoptosis was defined as the percentage of annexin V-positive cells. (b) Western blotting results of cytochrome c, cleaved caspase-9, and caspase-3 protein levels in KBM5 and KBM5-STI cells after Mdivi-1 treatment. ${ }^{* *} \mathrm{p}<0.01,{ }^{* * *} \mathrm{p}<0.001$.

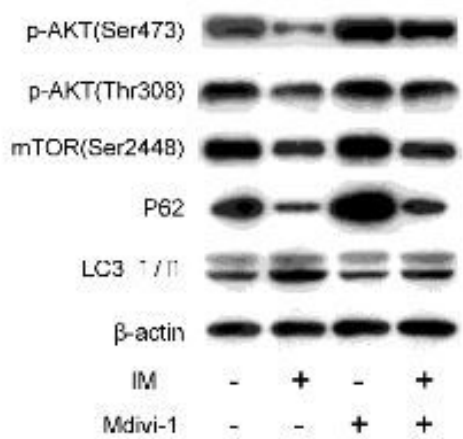

d

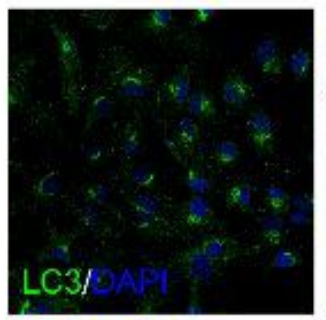

KBM5

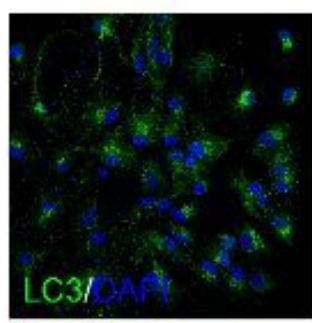

KBM5-STI

f

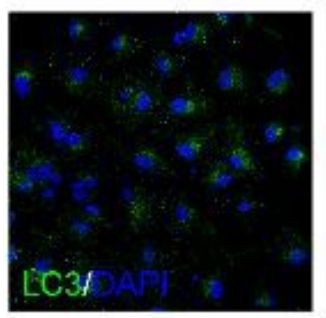

KBM5

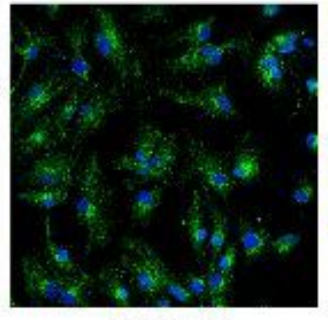

KBM5+IM

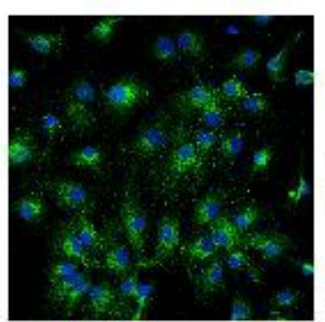

KBM5-STI+IM

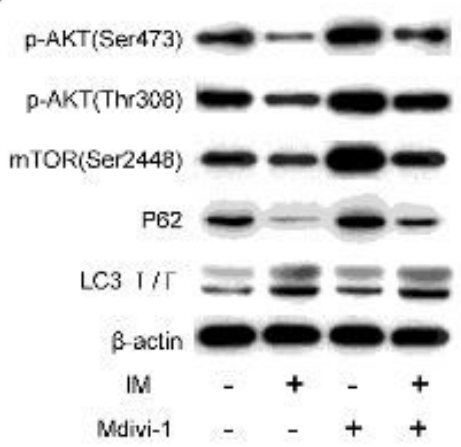

p-AKT(Ser473) p-AKT(Thr308) mTOR(Ser2448)
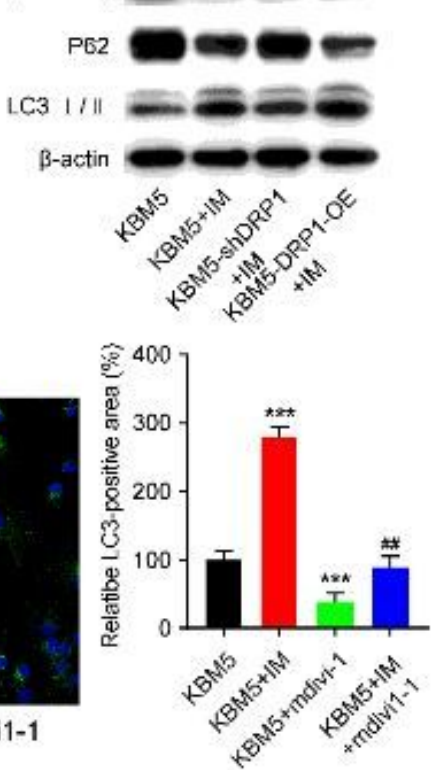

$50 \mathrm{\mu m}$

KBM5-STI+|M + Mdivi1-1

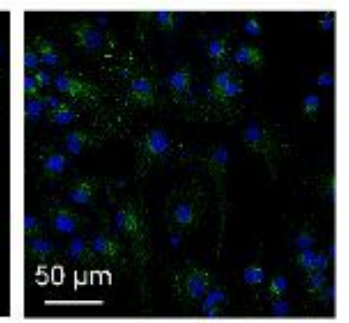

KBM5+|M +Mdivi1-1

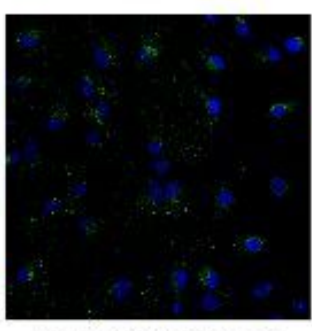

KBM5-STI+Mdivi-1

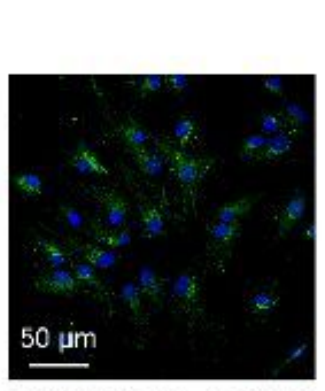

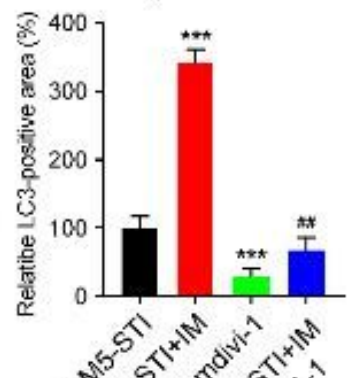
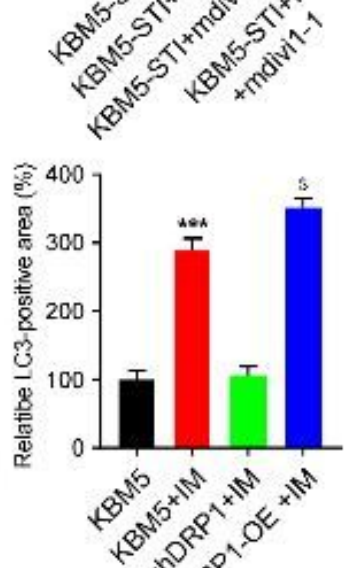

KBM5-DRP1-OE + IM

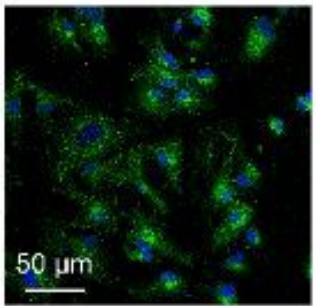

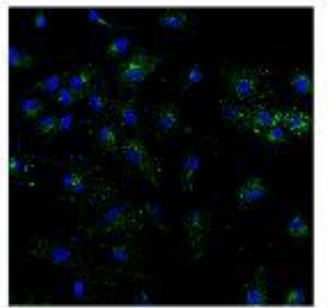

KBM5-shDRP1+IM

$\mathrm{KBM} 5+\mathrm{IM}$

\section{Figure 5}


Inhibition of DRP1 downregulates imatinib-induced autophagy in CML cells. (a, b, c) Western blotting analysis to evaluate the expression of autophagy-related proteins p-AKT(Ser473)『p-AKT(Thr308),

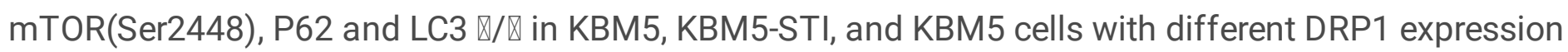
after imatinib and/or Mdivi-1 treatment. (d, e, f) Representative confocal microscopy images and relative LC3-positive area of above CML cell lines under imatinib and/or Mdivi-1 stimulation. Blue spots indicate nuclei stained with 4',6-diamidino-2-phenylindole (DAPI). Green spots show autophagic vacuoles stained with LC3B dye. $\$$ indicate $p<0.05$. \#\# indicate $p<0.01$. *** indicate $p<0.001$.

a

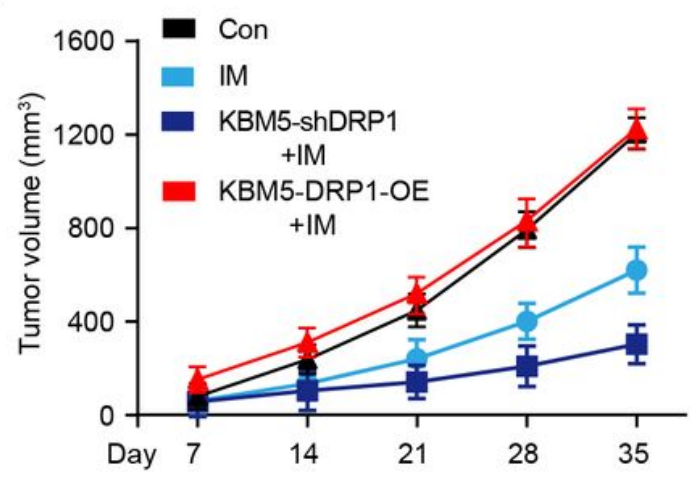

d

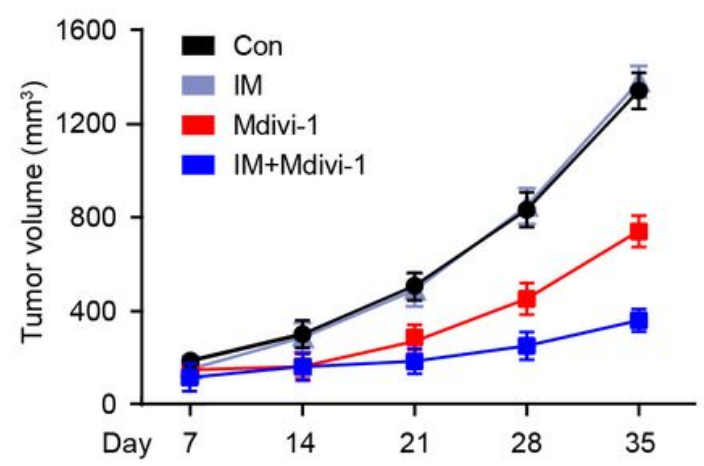

b

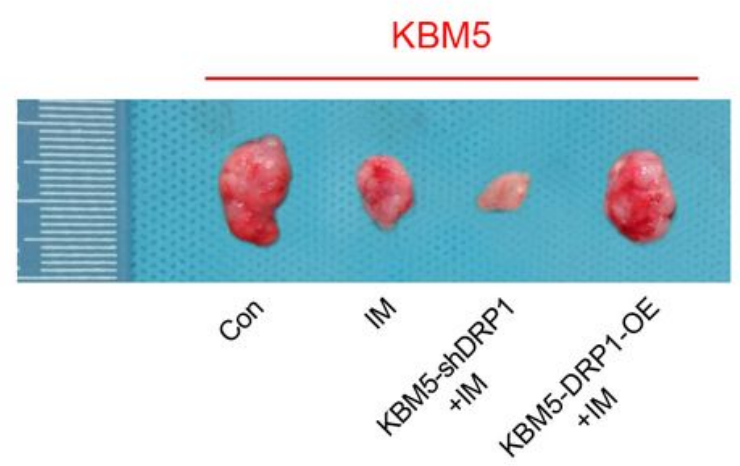

e

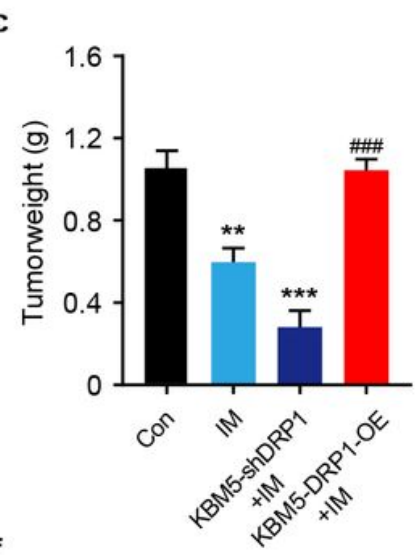

KBM5-STI
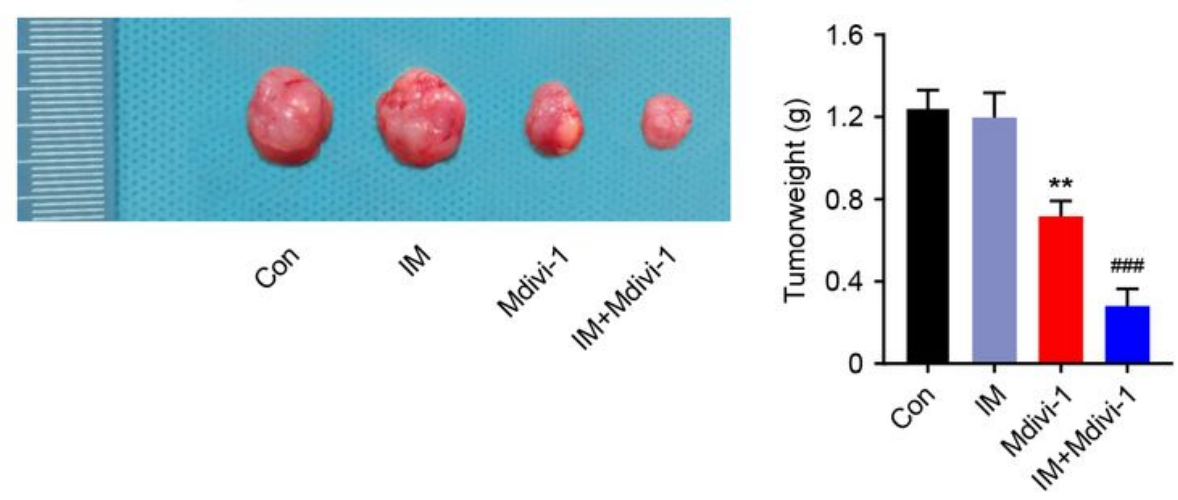

\section{Figure 6}

Inhibition of DRP1 induces decrease of tumor growth in imatinib-resistant CML cells in vivo. $(a, b, c)$ Analysis of tumor volume, size, and weight of KBM5 cells with different DRP1 expression under imatinib treatment. ( $d, e, f)$ Results of tumor volume, size, and weight of KBM5-STI with imatinib and/or midivi-1 treatment. $* \star$ indicate $p<0.01$. $* \star \star \rrbracket \# \# \#$ indicate $p<0.001$. 